\title{
CORRIGENDUM
}

\section{Metabolic targets for cancer therapy}

Lorenzo Galluzzi, Oliver Kepp, Matthew G. Vander Heiden and Guido Kroemer

Nature Reviews Drug Discovery 12, 829-846 (2013)

There were some inaccuracies in the following sentence on $\mathrm{p} 839$ of the article: "Along similar lines, the pharmacological or genetic inhibition of phosphoglycerate mutase 1 (PGAM1) reduces tumour growth in vitro and in vivo, perhaps owing (at least in part) to the G6PD-inhibitory effects of 3-phosphoglycerate ${ }^{222}$. That said, genetic defects that have an impact on the enzymatic activity of G6PD are common among individuals living in geographical areas with a history of endemic malaria ${ }^{223}$."

The correct sentence is as follows: "Along similar lines, the pharmacological or genetic inhibition of phosphoglycerate mutase 1 (PGAM1) reduces tumour growth in vitro and in vivo, perhaps owing (at least in part) to the PPP-inhibitory effects of 3-phosphoglycerate ${ }^{222}$. That said, genetic defects that have an impact on the enzymatic activity of G6PD (and hence inhibit the PPP) are common among individuals living in geographical areas with a history of endemic malaria ${ }^{223}$."

This has now been corrected in the online version of the article. 